\title{
MOOC y modelos de aprendizaje combinado. Una aproximación práctica
}

\section{(MOOCs and blended learning models. A practical approach)}

Teresa Torres-Coronas

María-Arántzazu Vidal-Blasco

Universitat Rovira i Virgili (España)

DOI: http://dx.doi.org/10.5944/ried.22.2.24093

\section{Cómo referenciar este artículo:}

Torres-Coronas, T., y Vidal-Blasco, M. A. (2019). MOOC y modelos de aprendizaje combinado. Una aproximación práctica. RIED. Revista Iberoamericana de Educación a Distancia, 22(2), pp. 325-343. doi: http://dx.doi.org/10.5944/ ried.22.2.24093

\section{Resumen}

Cada vez existe un mayor interés en analizar cómo los entornos virtuales influyen en el aprendizaje. En particular, los investigadores se centran en evaluar diseños educativos que combinan los MOOC con la enseñanza presencial. El presente artículo analiza una experiencia de aprendizaje mixto en una institución de educación superior española. Su objetivo es presentar un estudio de caso y discutir el enfoque estratégico para integrar un MOOC en estudios de grado presenciales. En el caso analizado, los estudiantes aprenden utilizando una combinación de clases presenciales con aprendizaje virtual. El objetivo es mejorar el rendimiento académico y reducir la tasa de abandono en estudiantes de primer curso. La estrategia de evaluación de esta experiencia se centra en la realización de cuestionarios y grupos focales para interpretar los resultados y la opinión de los diferentes grupos de interés. El análisis realizado sintetiza la opinión de las principales partes interesadas sujetas a estudio en la institución, esto es, la universidad, los estudiantes y el personal académico. Del estudio realizado se desprende que los modelos de aprendizaje combinado mejoran la calidad de la educación de los estudiantes, fortalecen su rendimiento académico y alientan al personal académico a innovar constantemente en su proceso didáctico.

Palabras clave: enseñanza superior; tecnologías de la información y de la comunicación; estudio de caso; métodos de enseñanza; calidad de la educación. 


\begin{abstract}
Interest is growing in analyzing how virtual environments affect the learning process. In particular, researchers focus on analyzing blended learning designs. These environments combine MOOC with face-to-face teaching. This article analyzes a blended learning experience in a Spanish higher education institution. Its aim is to present a case study and to discuss the strategic approach to integrate a MOOC in face-to-face undergraduate studies. In the analyzed case, on-campus students are taught using a combination of face-to-face classroom with online learning. The objective is to improve their academic performance and to reduce the drop-out rate among first-year students. The evaluation strategy of this experience focuses on conducting surveys and focus groups to interpret the results and the opinion of the different stakeholders, that is, the university, the students and the academic staff. The analysis shows that blended learning models improve the quality of students' education, strengthen students' academic performance, and encourage academic staff to constantly innovate in their didactic process.
\end{abstract}

Keywords: higher education, information and communication technologies; case study; teaching method; teaching quality.

La incorporación de los MOOC (cursos en línea masivos y abiertos o Massive Online Open Courses) a la educación superior ha cambiado la manera de adquirir conocimiento y mejorado la enseñanza. En 2014, la Universidad de Harvard comenzó a estudiar la posibilidad de incorporar los MOOC a las aulas siguiendo la línea de estudios que valoraban positivamente el aprendizaje en entornos digitales (Kovanović, Joksimović, Gašević, Siemens y Hatala, 2015) y la filosofía del conectivismo. En general, los MOOC ofrecen herramientas sociales para crear tanto comunidades de aprendizaje que se apoyan y asesoran mutuamente (Zhang, Huang, Wang y Cao, 2018) como entornos flexibles que encajen con participantes con objetivos y necesidades muy dispares. Para Clarke (2013), la escalabilidad es el atributo clave de los MOOC porque, una vez implantados, permiten alcanzar una audiencia global y, al mismo tiempo, llegar a audiencias más acotadas. Esta segunda opción de los MOOC es la que fusiona las ventajas de la tecnología y el aprendizaje autónomo con las de la interacción cara a cara en las aulas (Conijn, Vand den Beemt y Cuijpers, 2018; Yousef, Chatti y Schroeder, 2014).

La combinación de virtualidad con presencialidad es, en opinión de Zhang et al. (2018), un enfoque pedagógico completo. Este es el caso de la asignatura Orientación Profesional y Académica (OPA), pionera en la integración de los MOOC a nivel de grados universitarios. Esta asignatura tiene dos características que condicionan su diseño curricular. En primer lugar, la gran cantidad de estudiantes matriculados (desde el año 2015 se superan los doscientos estudiantes) y, en segundo lugar, el variado perfil de entrada de los estudiantes. Alrededor del 60\% son admitidos a través de la prueba de acceso a la universidad (PAU), mientras que el resto vienen de 
Formación Profesional 2 (FP2), Módulo Profesional 3 (MP3), Ciclos Formativos de Grado Superior (CFGS) o equivalente. Este perfil académico de entrada condiciona el rendimiento de los estudiantes y su tasa de abandono, que es mayor en los estudiantes de ciclos formativos. Estas dos variables -número de estudiantes y perfil de entrada- reforzaron la decisión de introducir el modelo de aprendizaje combinado (b-MOOC).

La tipología inicial de MOOC como un modelo pensado para la formación a lo largo de la vida ha evolucionado en los últimos años (Osuna-Acedo, Marta-Lazo y Frau-Meigs, 2018). Una de estas variantes son los b-MOOC, de los que Yousef, Chatti, Schroeder y Wosnitza (2015) recogen una de las primeras referencias de análisis de caso. En su propuesta, los estudiantes complementan las clases presenciales con contenido en línea y materiales en abierto. De esta manera, estudiantes matriculados en un grado oficial comparten un espacio de aprendizaje con participantes dispersos geográficamente que no están dentro de un sistema de formación reglada ni oficial (Ronkowitz y Ronkowitz, 2015). Se trata de una nueva pedagogía que combina el aula con un aprendizaje más auto-organizado y en red.

La literatura académica señala entre los objetivos del b-MOOC la reducción de las tasas de abandono y la mejora del rendimiento académico. Estos resultados se consiguen fusionando el aprendizaje virtual con el presencial (Gašević, Kovanović, Jokosimovic y Siemens, 2014; Holotescu, Grosseck, Cretu y Naaji, 2014; Israel, 2015). En este trabajo se presenta un caso de aplicación de este modelo combinado de aprendizaje a los estudiantes de la asignatura OPA que se imparte en el primer curso de los grados de ingeniería de una universidad española. El análisis compara la situación del curso 2015-16 (año de implantación del modelo) con la del curso 2018-19. Esta comparación longitudinal en el tiempo permite validar la idoneidad del modelo y verificar los resultados iniciales obtenidos en el curso 2015-16.

El siguiente apartado recoge la metodología y resultados, siguiendo una perspectiva sistémica que tiene en cuenta los tres principales grupos de interés: la Universidad, los estudiantes y el personal docente. De los resultados se desprende que los modelos de aprendizaje combinado, también denominados híbridos o mixtos (blended-learning), más allá de buscar una viabilidad económica de los MOOC al crear sinergias con el aula tradicional, impactan en la calidad de la oferta educativa, fortalecen el rendimiento académico y motivan al profesorado a seguir innovando.

\section{METODOLOGÍA Y RESULTADOS}

Este apartado recoge la metodología aplicada al análisis del caso presentado, así como la discusión y análisis de resultados de cada uno de los grupos de interés que forman parte del estudio. La metodología ha aplicado la técnica de grupos focales, así como el análisis de cuestionarios autoadministrados.

Para analizar el papel institucional en el fomento de modelos mixtos de aprendizaje se realizaron dos grupos focales con cinco responsables académicos. Se 
consideraron las dimensiones de Jansen y Schuwer (2015) y se discutió cómo integrar el espíritu de los MOOC en la clase tradicional. El primer grupo focal pertenece al curso 2015-16 y, el segundo al curso 2018-19. Las opiniones se presentan en el texto indicando el grupo focal al que pertenecen. De esta manera, el experto 1 del grupo focal 1 queda recogido en el texto como "exp.1, grupo 1", y así sucesivamente.

Para recoger la opinión de los estudiantes se siguió la metodología de Yousef, Chatti, Schroeder y Wosnitza (2015). Estos autores evalúan los modelos de enseñanza a partir de ocho dimensiones: el aprendizaje combinado, la flexibilidad, el diseño instruccional y las metodologías de aprendizaje, el contenido de alta calidad, el aprendizaje a lo largo de toda la vida, el aprendizaje en red, la apertura y el aprendizaje focalizado en el estudiante. En el presente artículo se recogen los resultados de las tres primeras dimensiones partiendo de datos primarios cuantitativos. Para ello se suministró un cuestionario en línea a la totalidad de estudiantes de la asignatura. En el curso 2015-16, la asignatura contaba con 192 estudiantes (con un 10,9\% de mujeres), siendo la tasa de participación final del 25,4\%. Por su parte, en el curso 2018-19 la asignatura tenía un total de 296 (13,5\% mujeres) y la tasa de participación fue del $31,3 \%$. En ambos cuestionarios se aplicó una escala tipo Likert de cinco niveles, correspondiendo 1 a "Totalmente en desacuerdo" y 5 a "Totalmente de acuerdo". Los resultados de estos cuestionarios se presentan de acuerdo al análisis de escalas ordinales de Norman (2010).

Finalmente, se analiza el papel del personal docente en un proceso que comienza con el diseño del curso, continúa con la prueba piloto y culmina con la evaluación del proceso de implantación desde el curso 2015-16 al 2018-19. El análisis sintetiza las opiniones expresadas por los tres profesores del curso, en dos grupos focales diferentes (uno correspondiente al curso 2015-16 y otro al curso 2018-19). El profesor 1 del grupo focal 1 queda recogido en el texto como "prof.1, grupo 1". La discusión de resultados se presenta a modo de storytelling.

\section{Análisis desde la dimensión institucional}

Como ponen de manifiesto Baker, Bujak y DeMillo (2012), "las instituciones efectivas son aquellas que exploran de manera proactiva enfoques innovadores para reestructurar la entrega de 'contenido' junto con el cambio institucional" (p. 330). Estos enfoques incluyen modelos de aprendizaje combinado y una profunda comprensión de los beneficios y las consecuencias de implementar soluciones tecnológicas que mejoren la práctica actual, sean compatibles con los valores existentes y generen resultados observables. Estos cambios no son factibles sin instituciones que impulsen la alfabetización digital del profesorado (Mirriahi, Alonzo, McIntyre, Kligyte y Fox, 2015). Evidentemente, este proceso tiene un coste en términos de recursos económicos y tiempo del personal docente.

El estudio presentado utiliza el enfoque de Jansen y Schuwer (2015) para analizar la perspectiva institucional, identificar las fuerzas impulsoras del cambio 
y determinar cómo puede ajustarse el b-MOOC a la estrategia institucional de la universidad. El Plan director de la institución de educación superior analizada, aprobado por el Claustro universitario en 2004, contiene las pautas sobre los modelos de educación virtual, los métodos de enseñanza y evaluación y las competencias transversales. Estas políticas institucionales son las que deben guiar la actuación docente y el fomento de la integración de la tecnología en el diseño curricular (Garrison y Vaughan, 2011).

El Plan de la Universidad analizada establece que "la ordenación académica se tiene que implementar de forma que se asegure el logro de los resultados propuestos en cada uno de los planes de formación y su calidad, mediante estrategias que impliquen revisión del mapa competencial, el posible modelo de virtualización y la revisión de metodologías docentes y de evaluación" (Plan director para reestructurar la oferta docente de grado, máster y doctorado de la Universidad, Claustro de 4 de junio de 2015). En este sentido, cuando parte del equipo docente decide poner en marcha un modelo mixto de aprendizaje (b-MOOC) debe hacerlo valorando su contribución a la estrategia descrita en el Plan Director y tener en cuenta cómo va a impactar en la innovación docente, la mejora de resultados académicos y el fomento de la investigación en innovación docente. Hollands y Tirthali (2014) consideran estos tres factores como las fuerzas impulsoras del cambio.

$\mathrm{El}$ análisis de los grupos focales permite ver un primer resultado que se mantiene a lo largo del período analizado. En contraposición al estudio de Jansen y Schuwer (2015), ninguno de los dos grupos considera relevantes factores como la captación de estudiantes o la mejora de la visibilidad de la universidad como factores que la institución utiliza para impulsar los MOOC. Se considera más bien que cuando el MOOC se integra en el aula tradicional se convierte en una herramienta de apoyo y de flexibilización de la formación presencial. Y aquí es donde aparece su valor.

Desde el punto de vista de apoyo a la formación presencial "el b-MOOC no conlleva solo a hacer que los materiales docentes estén disponibles en línea. Es una innovación disruptiva del proceso de enseñanza-aprendizaje” (exp.1, grupo 1). Esta línea argumental también se mantiene en los dos períodos analizados. "Es posible que los MOOC, por las altas tasas de abandono, no hayan tenido el éxito esperado. Esto no impide que su integración en el aula tradicional pueda beneficiar el proceso de enseñanza-aprendizaje tradicional” (exp.2, grupo 2).

Un MOOC es un "curso" y, en consecuencia, debería ofrecer una experiencia global (Jansen y Schuwer, 2015). El b-MOOC proporciona nuevas experiencias de aprendizaje (Bralić y Divjak, 2018). Al igual que los MOOC es "una entidad en sí mismo. Solo así se pueden poner en marcha pedagogías innovadoras en el aprendizaje virtual que no olviden ni el contenido educativo ni la interacción ni la inclusión de actividades de aprendizaje y evaluativas adaptadas con su correspondiente guía de estudio" (exp.5, grupo 1). Sin duda, "debe añadir valor a la oferta curricular" (exp.2, grupo 1). Y, como modelo que utiliza un entorno virtual el b-MOOC "sirve de apoyo 
al desarrollo de competencias digitales, unas competencias transversales que todos los planes curriculares deberían incluir" (exp.4, grupo 2).

De igual manera, existe consenso a la hora de afirmar que "la hibridación es una oportunidad para introducir flexibilidad en el proceso de aprendizaje y permitir a los estudiantes vivir una experiencia diferente a la de la docencia presencial" (exp.5, grupo 1). En el caso de personas con diversidad funcional, "la virtualidad puede facilitarles el correcto seguimiento de un curso, al no exigir tanta presencia física en el aula" (exp.2, grupo 1). Sin embargo, "estos entornos no siempre están correctamente adaptados a otros tipos de diversidad, como la cognitiva" (exp.5, grupo 2). En ambos grupos focales existe consenso a la hora de afirmar que los modelos mixtos juegan un papel en la vida del estudiante, permitiéndole adaptar mejor su horario.

Desde el punto de vista del desarrollo profesional y, aunque "no todos los docentes tienen las competencias digitales necesarias para aplicar de manera innovadora un modelo de aprendizaje híbrido" (exp.3, grupo 2), el b-MOOC es "una forma eficaz de repensar y aprender sobre la enseñanza en línea" (exp.4, grupo 1). Por otra parte, y a nivel estratégico, "elaborar el contenido de un MOOC es costoso en tiempo y recursos, con lo que si se quiere reducir el riesgo y fomentar su uso deben buscarse sinergias entre la clase tradicional y la virtual" (exp.1, grupo 1). Para la Universidad, y en la línea con lo expuesto por Yuan, Powell y Olivier (2014), el b-MOOC representa una estrategia relacionada con la mejora de la oferta educativa, una manera de desarrollar las competencias digitales de los estudiantes y una puerta a aumentar la satisfacción de aquellos que prefieren experiencias de aprendizaje con un componente más tecnológico (Mirriahi, Alonzo y Fox, 2015). El "b-MOOC se adapta mucho mejor a las necesidades y preferencias del estudiante actual" (exp.2, grupo 2) y, puede llegar a "impactar positivamente en su nivel de motivación" (exp.1, grupo 1; exp.4, grupo 2).

Sin embargo, "los modelos b-MOOC y de aprendizaje combinado no serán una realidad sin apoyo técnico" (exp.4, grupo 1) puesto que, más allá de las horas de dedicación del propio docente, se necesita que "la universidad dedique recursos y apoyo de personal técnico para asistir la labor del personal docente" (exp.1, grupo 2).

\section{Análisis desde la dimensión de los estudiantes}

El modelo b-MOOC es la combinación de dos modelos de enseñanzaaprendizaje: enseñanza tradicional e innovación tecnológica. En la asignatura de OPA los estudiantes asisten a clases presenciales durante las primeras diez semanas del semestre. Las cinco semanas restantes aprenden a través de un entorno virtual abierto.

La perspectiva del estudiante se analizó a través de un cuestionario y, en paralelo, se evaluaron indicadores objetivos como la tasa de éxito (relación entre los créditos aprobados sobre los créditos presentados), la tasa de rendimiento (créditos 
aprobados sobre matriculados) y la tasa de abandono (créditos presentados sobre matriculados) de los cursos 2015-16 y 2018-19.

\section{Análisis de la percepción del alumnado}

La aceptación por parte del estudiante es una variable importante para determinar el éxito de cualquier experiencia de aprendizaje. En el caso de los modelos híbridos, la aceptación es posible si el sistema es fácil de usar, aplicable y útil (Tselios, Daskalakis y Papadopoulou, 2011). Para el estudiante, los modelos híbridos presentan retos añadidos en lo relativo a la evaluación de resultados, dado que la interacción virtual y la presencial puede complicar la percepción sobre su experiencia de aprendizaje.

Los valores registrados (tabla 1) fueron altos en todas las dimensiones: mejora del desempeño académico, motivación, satisfacción de los estudiantes y efectividad del proceso de aprendizaje. En las preguntas 2 a 4 y en el último período analizado se observa un ligero retroceso en el nivel de satisfacción.

Tabla 1. Aprendizaje combinado y desempeño académico. Percepción del alumnado

\begin{tabular}{|c|c|c|c|}
\hline & \multirow{2}{*}{ 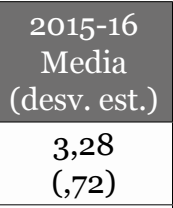 } & \multirow{2}{*}{$\begin{array}{c}\begin{array}{c}2018-19 \\
\text { Media }\end{array} \\
\text { (desv. est.) } \\
3,86 \\
(1,35)\end{array}$} \\
\hline 1 & $\begin{array}{l}\text { Combinar el aprendizaje presencial y virtual me ayuda a } \\
\text { mejorar mis resultados académicos. }\end{array}$ & & \\
\hline 2 & $\begin{array}{l}\text { Combinar el aprendizaje presencial y virtual aumenta mi } \\
\text { motivación para compartir y descubrir nuevas ideas. }\end{array}$ & $\begin{array}{l}4,58 \\
(, 25)\end{array}$ & $\begin{array}{l}4,13 \\
(, 79)\end{array}$ \\
\hline 3 & $\begin{array}{l}\text { Combinar el aprendizaje presencial y virtual me permite } \\
\text { realizar tareas más rápidamente. }\end{array}$ & $\begin{array}{l}4,10 \\
(, 64)\end{array}$ & $\begin{array}{r}3,45 \\
(1,54)\end{array}$ \\
\hline \multirow[t]{2}{*}{4} & $\begin{array}{l}\text { El enfoque de aprendizaje combinado se puede utilizar para } \\
\text { complementar el enfoque tradicional en el aula. }\end{array}$ & $\begin{array}{l}4,40 \\
(, 57)\end{array}$ & $\begin{array}{l}3,89 \\
(, 87)\end{array}$ \\
\hline & Alfa de Cronbach &, 76 & ,77 \\
\hline
\end{tabular}

Fuente: Elaboración propia

El b-MOOC permite al estudiante aprender a su ritmo y acceder a los materiales de una manera más flexible. En la tabla 2, se constata que el entorno dota al estudiante de la flexibilidad buscada. Y, tal y como corroboran estudios previos como el de Nazarenko (2015), la satisfacción con el modelo combinado es alta. 
Tabla 2. Aprendizaje combinado y flexibilidad. Percepción del alumnado

\begin{tabular}{|c|c|c|c|}
\hline & & $\begin{array}{c}\text { 2015-16 } \\
\text { Media } \\
\text { (desv. est.) }\end{array}$ & $\begin{array}{c}\text { 2018-19 } \\
\text { Media } \\
\text { (desv. est.) }\end{array}$ \\
\hline 1 & $\begin{array}{l}\text { Puedo acceder a las actividades de aprendizaje en cualquier } \\
\text { momento que me convenga. }\end{array}$ & $\begin{array}{l}4,50 \\
(, 64)\end{array}$ & $\begin{array}{l}4,00 \\
(, 85)\end{array}$ \\
\hline 2 & $\begin{array}{l}\text { Puedo acceder al contenido del temario y a las actividades de } \\
\text { aprendizaje desde cualquier lugar. }\end{array}$ & $\begin{array}{l}4,33 \\
(, 69)\end{array}$ & $\begin{array}{l}4,50 \\
(, 35)\end{array}$ \\
\hline 3 & $\begin{array}{l}\text { El entorno de aprendizaje me proporciona una gran variedad } \\
\text { de materiales entre los que elegir. }\end{array}$ & $\begin{array}{l}4,18 \\
(, 78)\end{array}$ & $\begin{array}{l}3,75 \\
(1,5)\end{array}$ \\
\hline 4 & Puedo acceder a los materiales de aprendizaje sin dificultad. & $\begin{array}{l}4,28 \\
(, 65)\end{array}$ & $\begin{array}{l}4,08 \\
(, 85)\end{array}$ \\
\hline 5 & $\begin{array}{l}\text { La guía de contenido me lleva a explorar el curso más a } \\
\text { fondo. }\end{array}$ & $\begin{array}{l}4,10 \\
(, 75)\end{array}$ & $\begin{array}{l}4,06 \\
(, 85)\end{array}$ \\
\hline \multirow[t]{2}{*}{6} & $\begin{array}{l}\text { El entorno de aprendizaje me permite centrarme en las } \\
\text { actividades de aprendizaje que son adecuadas para mí. }\end{array}$ & $\begin{array}{l}4,13 \\
(, 67)\end{array}$ & $\begin{array}{c}3,77 \\
(1,23)\end{array}$ \\
\hline & Alfa de Cronbach & ,82 & ,79 \\
\hline
\end{tabular}

Fuente: Elaboración propia

Según Margaryan, Bianco y Littlejohn (2015, p. 78), "la calidad del diseño instruccional de un curso es un indicador crítico y un requisito previo sobre el potencial del curso para un aprendizaje efectivo". En relación a esta variable (tabla 3), los valores más bajos corresponden a ítems relacionados con la rapidez de respuesta del profesorado y la retroalimentación recibida. Estos resultados son consistentes con un diseño curricular escalable que intenta reducir el tiempo de dedicación del profesorado durante la impartición de la parte virtual del curso. Sin embargo, también refleja la necesidad de que el equipo docente tenga un rol activo facilitando y guiando al estudiante en el proceso de adquisición de nuevo conocimiento (Zhou, 2016).

Tabla 3. Diseño institucional y metodología de aprendizaje. Percepción del alumnado

\begin{tabular}{|c|c|c|c|}
\hline & & $\begin{array}{c}\text { 2015-16 } \\
\text { Media } \\
\text { (desv. est.) }\end{array}$ & $\begin{array}{c}\text { 2018-19 } \\
\text { Media } \\
\text { (desv. est.) }\end{array}$ \\
\hline 1 & $\begin{array}{l}\text { Los objetivos de aprendizaje están claramente establecidos } \\
\text { en cada tema. }\end{array}$ & $\begin{array}{l}4,20 \\
(, 65)\end{array}$ & $\begin{array}{c}3,86 \\
(1,53)\end{array}$ \\
\hline 2 & El ámbito de cada tema está claramente establecido. & $\begin{array}{l}4,03 \\
(, 80)\end{array}$ & $\begin{array}{l}4,13 \\
(, 71)\end{array}$ \\
\hline 3 & $\begin{array}{l}\text { La estructura de este curso me mantiene centrado en lo que } \\
\text { se debe aprender. }\end{array}$ & $\begin{array}{l}3,55 \\
(, 69)\end{array}$ & $\begin{array}{l}3,85 \\
(, 75)\end{array}$ \\
\hline
\end{tabular}




\begin{tabular}{|c|c|c|c|}
\hline & \multirow{2}{*}{$\begin{array}{c}\begin{array}{c}2015-16 \\
\text { Media } \\
\text { (desv. est.) }\end{array} \\
4,10 \\
(, 78)\end{array}$} & \multirow{2}{*}{ 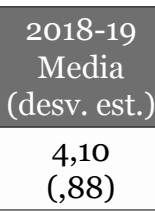 } \\
\hline 4 & Siempre sé dónde estoy en el curso. & & \\
\hline 5 & $\begin{array}{l}\text { Las diversas herramientas del entorno de aprendizaje son } \\
\text { efectivas. }\end{array}$ & $\begin{array}{l}4,23 \\
(, 83)\end{array}$ & $\begin{array}{c}3,23 \\
(1,89)\end{array}$ \\
\hline 6 & $\begin{array}{l}\text { Tengo la posibilidad de preguntarle a mi tutor/a lo que no } \\
\text { entiendo. }\end{array}$ & $\begin{array}{l}4,23 \\
(, 80)\end{array}$ & $\begin{array}{l}4,31 \\
(, 66)\end{array}$ \\
\hline 7 & Los/as tutores/as responden rápidamente a mis consultas. & $\begin{array}{c}3,87 \\
(1,02) \\
\end{array}$ & $\begin{array}{c}3,59 \\
(1,34)\end{array}$ \\
\hline 8 & Puedo acercarme al equipo docente cuando lo necesito. & $\begin{array}{l}4,08 \\
(, 83)\end{array}$ & $\begin{array}{l}4,13 \\
(, 73) \\
\end{array}$ \\
\hline 9 & $\begin{array}{l}\text { El sistema de evaluación de este curso mejora mi proceso de } \\
\text { aprendizaje. }\end{array}$ & $\begin{array}{l}3,93 \\
(, 85)\end{array}$ & $\begin{array}{l}4,01 \\
(, 57)\end{array}$ \\
\hline 10 & $\begin{array}{l}\text { Los/as tutores/as me envían retroalimentación completa } \\
\text { sobre mi tarea. }\end{array}$ & $\begin{array}{l}3,83 \\
(, 91)\end{array}$ & $\begin{array}{c}3,75 \\
(1,46)\end{array}$ \\
\hline \multirow[t]{2}{*}{11} & $\begin{array}{l}\text { Los criterios de calificación se comunicaron claramente al } \\
\text { inicio del curso. }\end{array}$ & $\begin{array}{l}4,04 \\
(, 79)\end{array}$ & $\begin{array}{c}4,13 \\
(, 53)\end{array}$ \\
\hline & Alfa de Cronbach & ,83 & 81 \\
\hline
\end{tabular}

Fuente: Elaboración propia

Con el modelo combinado se amplían los espacios y las oportunidades disponibles para el aprendizaje, la interacción y la colaboración y, para el profesorado se abren nuevas herramientas de apoyo a la gestión del curso relacionadas con la comunicación, evaluación y retroalimentación. Por tanto, no se trata solo de utilizar la tecnología para seguir una moda sino para ayudar a los estudiantes a lograr los objetivos de aprendizaje de manera más eficiente. La experiencia demuestra que el valor añadido de las clases magistrales puede complementarse con un entorno virtual que permite aprender de una manera más colaborativa, flexible y adaptada al ritmo de aprendizaje de cada persona.

\section{Análisis de indicadores}

Los estudiantes, especialmente los de primer curso, valoran formar parte de una comunidad presencial de aprendizaje (Caufield, Collier y Halawa, 2013; Owston, York y Murtha, 2013). En los modelos mixtos, la combinación del aula con el entorno virtual crea más cohesión social al fusionar la interacción presencial y virtual. Esto puede aumentar la tasa de éxito y, como se aprecia en las figuras 1 a 3, también reduce la tasa de abandono. El trabajo de Moskal, Dziuban y Hartman (2013) corrobora este último resultado. 
Los logros de este modelo híbrido son consistentes con los resultados de la investigación de Garrison y Anderson (2011). Para estos autores, la experiencia educativa de un estudiante depende de tres tipos de presencia: presencia cognitiva (o forma en que los estudiantes se involucran con el contenido), presencia social (o cómo los estudiantes se relacionan entre sí) y, presencia docente (o cómo los profesores se implican en el curso).

Desarrollar la interacción social es esencial en el diseño instruccional. Se requiere presencia social para generar confianza entre los estudiantes y que puedan, de esta manera, participar cómodamente en niveles más profundos de construcción de conocimiento social. Los modelos combinados crean más oportunidades para que los estudiantes se conecten con el contenido, con sus compañeros y con el profesorado.

La asignatura de OPA se implantó por primera vez en el año 2010, siguiendo un modelo totalmente presencial, siendo su tasa de éxito y rendimiento superior a la media del resto de asignaturas de primer curso de los grados de las titulaciones analizadas. Sin embargo, en algunas titulaciones en las que se impartía la asignatura, como el Grado en Ingeniería Eléctrica (GEE), los resultados del período 2010-2015 no habían sido tan satisfactorios debido al perfil del estudiante de nueva entrada. En los estudiantes de GEE que venían de ciclos formativos, y que constituían alrededor del 50\% del estudiante de nueva entrada, las tasas de abandono eran mayores y las tasas de éxito más reducidas. Para solucionar este problema, en el curso 2015-16 se decide integrar un MOOC con el aula tradicional.

Las figuras 1 a 3 muestran la evolución de la tasa de éxito, de rendimiento y de abandono del curso académico 2015-16 y 2018-19. Los porcentajes se muestran desglosados por titulación o grado: GEE, Grado en Ingeniería Electrónica Industrial y Automática (GEEiA), Grado en Ingeniería Informática (GEI) y el doble grado GEE+GEEiA. 
T. TORRES-Coronas; M. A. VIDAL-Blasco

MOOC Y MODELOS DE APRENDIZAJE COMBINADO. UNA APROXIMACIÓN PRÁCTICA

Figura 1. Tasa de éxito por titulación en los cursos 2015-16 y 2018-19

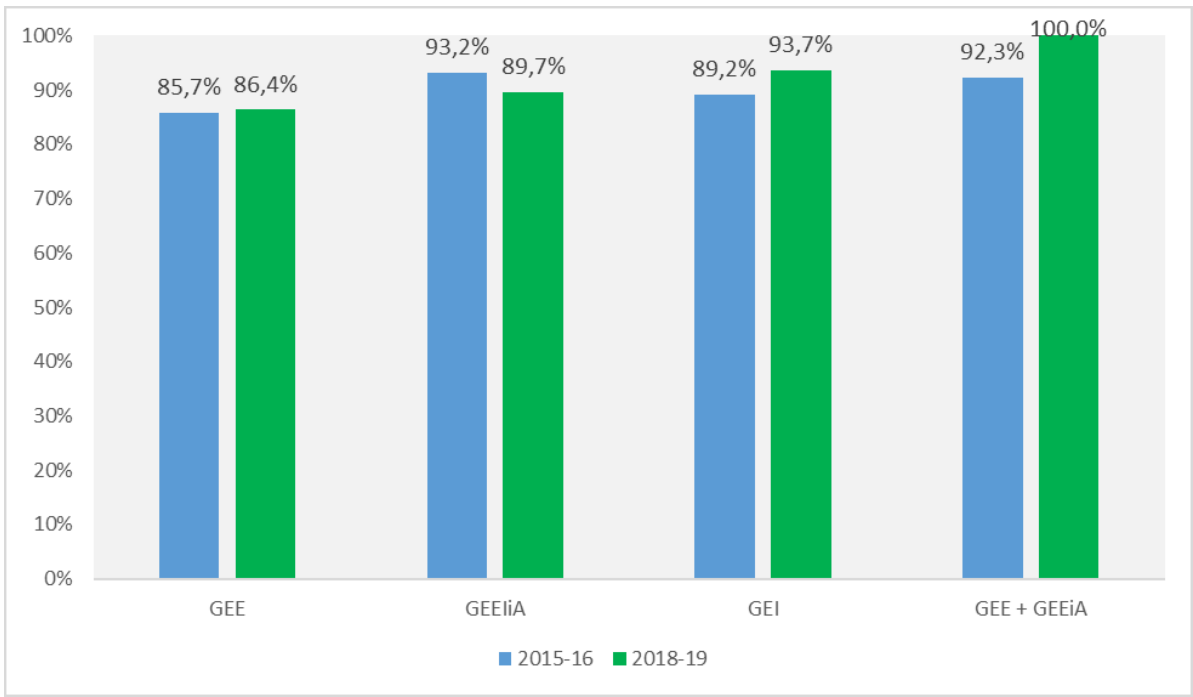

Figura 2. Tasa de rendimiento académico en los cursos 2015-16 y 2018-19

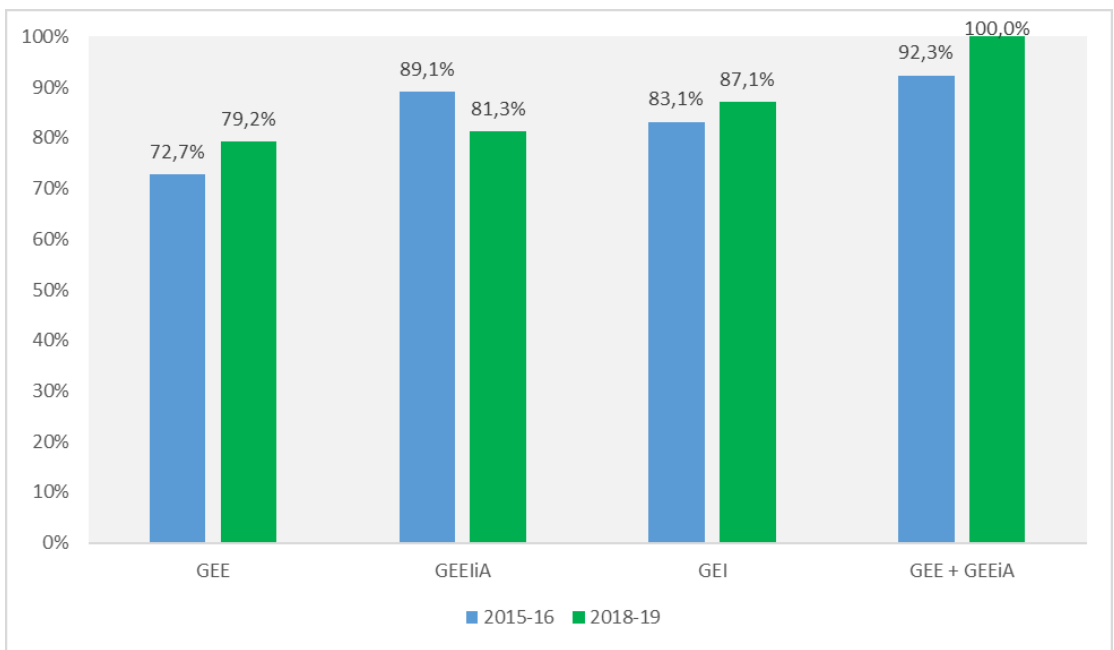


Figura 3. Tasa de abandono en los cursos 2015-16 y 2018-19

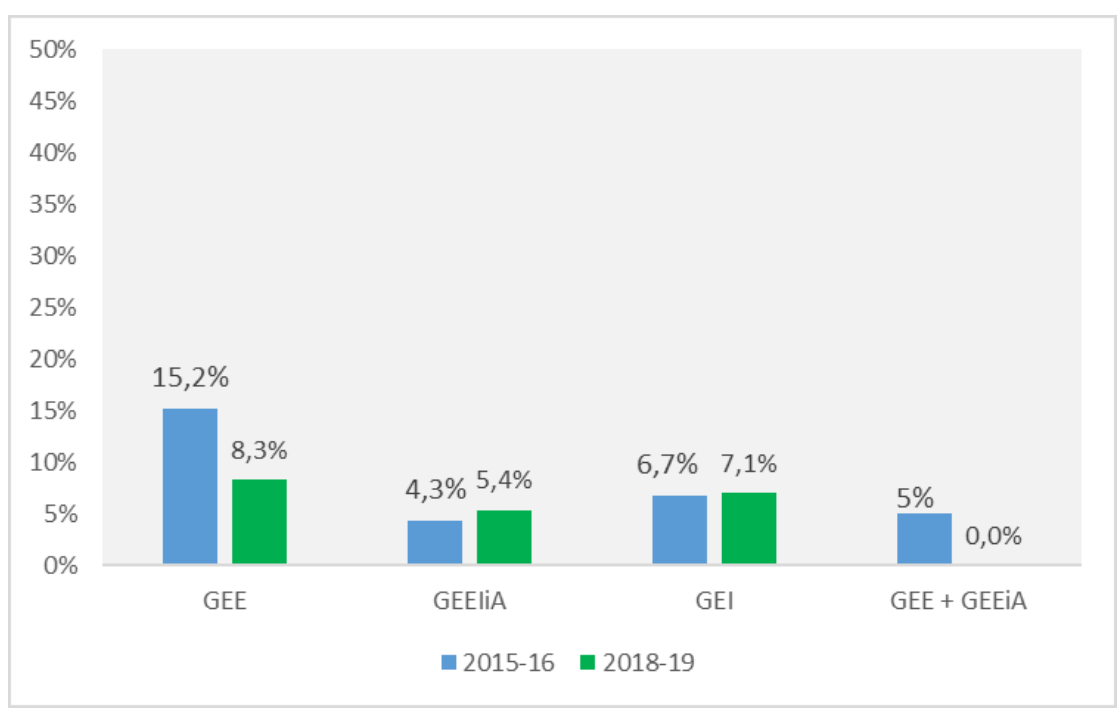

Los datos recogidos en las figuras anteriores permiten constatar cómo la introducción del aprendizaje combinado mejora las tres ratios, apreciándose la mayor caída en la tasa de abandono. El diseño del curso, altamente estructurado y pautado, ha sido uno de los motivos del éxito, tal y como también demuestran Banerjee y Duflo (2014), Yousef et al. (2015) y Loya, Gopal, Shukla, Jermann y Tormey (2015). Los estudiantes con un enfoque más flexible y que programan mejor su aprendizaje tienen, por regla general, más probabilidades de finalizar el curso. Los modelos mixtos guían mejor a los estudiantes que no son tan organizados, lo que ayuda a reducir la tasa de abandono.

En las titulaciones con tasas más altas de abandono y resultados académicos menos satisfactorios, el modelo mixto equilibra la tasa de éxito entre titulaciones, a pesar de que la tasa de rendimiento académico (figura 2) y la de abandono (figura 3) continúan siendo peores en el GEE. En conclusión, se puede afirmar que el b-MOOC se adecúa a los diferentes perfiles académicos de entrada del estudiante y mejora los resultados allí donde los datos históricos no eran tan satisfactorios.

\section{Análisis desde la dimensión del profesorado}

La última dimensión de análisis se centra en el rol del profesor en el diseño e implantación inicial de un entorno de aprendizaje combinado. En general, las razones por las que el personal docente puede ser reacio a adoptar tecnología educativa van desde la percepción de la relevancia de la tecnología para el aprendizaje de los 
estudiantes (Kennedy, Jones, Chambers y Peacock, 2011) a la falta de familiaridad con las herramientas (Handal, MacNish y Petocz, 2013) que se relaciona especialmente con el nivel de competencia digital del profesorado.

\section{Diseño del curso}

Las tareas clave en el desarrollo de un curso virtual en abierto y masivo incluyen el diseño del contenido (qué enseñar), el diseño del método de aprendizaje (o la selección de lecturas, vídeos educativos y contenido web), la configuración de las actividades de aprendizaje individuales y grupales, la fijación del calendario para las actividades, y la preparación de directrices sobre cómo utilizar el entorno con eficacia (etiqueta de red). A nivel pedagógico, la mayoría de experiencias de aprendizaje virtual siguen el conectivismo (Smith y Eng, 2013).

Por regla general, el conectivismo tiene como objetivo generar conocimiento a través de la interacción y ve el aprendizaje como un proceso de formación de redes. En el b-MOOC, esta tarea se hace más complicada por la necesidad de "hacer coincidir la parte virtual del curso con la presencial" (prof.2, grupo 1). Además, en opinión de un profesor, "aunque las tareas son esencialmente las mismas, los conocimientos y las competencias que deben aplicarse no lo son, ya que el diseño del curso requiere más tiempo y un mayor esfuerzo" (prof.1, grupo 1). Se necesita contar tanto con el apoyo de personal técnico para la adaptación de materiales a entornos virtuales como con recursos materiales (como equipos de grabación) o programas de edición de video. "Este apoyo no siempre es gratuito, por lo que si no hay recursos financieros disponibles el b-MOOC será imposible de realizar" (prof.3, grupo 1). Sin embargo, las dificultades no deben impedir que "los cursos sean motivadores y efectivos desde el punto de vista pedagógico" (prof.2, grupo 1). "La tecnología como medio para brindar una oportunidad de alcanzar objetivos de aprendizaje debe ser fácil de usar" (prof.1, grupo 1).

La aplicación del b-MOOC durante más de un curso académico puede permitir mejorar la eficacia del diseño, aunque supone más tiempo por parte del equipo docente. "Sin duda, a los cambios a hacer en la parte tradicional de una asignatura, en el modelo mixto, hay que añadir muchas horas para revisar el encaje con la parte virtual" (prof.2, grupo 2).

\section{Prueba piloto}

Normalmente no se hacen pruebas piloto en un curso presencial. Esto no es así en el diseño de cursos virtuales, donde "los materiales se deben testar, las actividades de evaluación funcionar correctamente y la tecnología no debe fallar para que el estudiante disfrute de la experiencia de aprendizaje" (prof.1, grupo 1). Este proceso implica más dedicación y recursos. Las pruebas piloto aunque no siempre reproducen 
las mismas condiciones que el curso final, sí permiten "realizar ajustes que si no se hicieran podrían conducir al fracaso del modelo" (prof.2, grupo 1).

\section{Impartición}

Para Coppola, Hiltz y Rotter (2002), las tareas de un profesor virtual se centran en responder preguntas y ayudar a los estudiantes a interaccionar con la información (dimensión cognitiva), gestionar la construcción de relaciones con los estudiantes (dimensión afectiva) y estructurar la interacción, motivar, coordinar la participación y evaluar los resultados (dimensión de gestión).

Uno de los problemas de los MOOC es que "las interacciones de los estudiantes con los instructores y otros participantes en el curso son necesariamente limitadas y menos integradoras que las interacciones en persona" (Hoxby, 2014, p. 530). Este problema se soluciona en el b-MOOC porque "la interacción se complementa con tutorías presenciales y unos lazos de confianza previos" (prof.1, grupo 1).

$\mathrm{Al}$ mismo tiempo, los profesores no deberían asumir que los alumnos saben cómo interactuar de manera virtual o cómo ser más responsables durante la parte de aprendizaje que se lleva a cabo en el entorno virtual. El equipo docente es responsable de conseguir que los estudiantes desarrollen las habilidades para involucrarse con el contenido del curso porque, "no se observa una mayor familiarización de los MOOC con el paso del tiempo ni mayores competencias digitales en los nuevos estudiantes que llegan a la universidad" (prof.3, grupo 2).

En los MOOC, "el aprendizaje se mejora mediante la participación tanto en la creación y el intercambio de contribuciones personales, como en las interacciones con las contribuciones de otros, pero la participación es voluntaria" (Baturay, 2015, p. 428). Esto "añade valor al b-MOOC porque los estudiantes interactúan con personas de todo el mundo" (prof.3, grupo 1) aunque, "es difícil repartir la carga docente para que los estudiantes tengan el tiempo suficiente de aprovechar todos los recursos e interacciones a su alcance" (prof.2, grupo 2).

El contexto influye en el aprendizaje (Hood, Littlejohn y Milligan, 2015; Liyanagunawardena, Lundqvist y Williams, 2015) y es el que permite "ofrecer oportunidades de aprendizaje y mejorar las notas a participantes con diferentes procedencias y disparidad en conocimientos" (prof.3, grupo 1). Esto solo es posible si "los recursos multimedia se diseñan para ayudar a los estudiantes a adquirir conocimiento y no solo para cubrir el expediente" (prof.2, grupo 1). Una vez conocido el perfil del estudiante, "el rediseño es una tarea necesaria para adecuar los perfiles al entorno de aprendizaje” (prof.2, grupo 2).

El altruismo es uno de los motivos por los que el profesorado participa en procesos de innovación docente (Hew y Cheung, 2014). Por ello, es importante que el profesorado reciba alguna compensación. El tiempo es un recurso limitado y el esfuerzo dedicado al b-MOOC va en detrimento de otras actividades. "Si la institución tiene un modelo que no premia este tipo de dedicación, el voluntarismo 
irá en detrimento del desarrollo profesional. La productividad, medida en términos de publicaciones, disminuirá" (prof.2, grupo 1). Una idea que persiste en el tiempo es que la innovación docente tiene un coste de oportunidad.

\section{CONCLUSIONES}

Las universidades, como ponen de manifiesto Torrisi-Steele y Drew (2013), se enfrentan al desafío de ofrecer experiencias de aprendizaje rentables y de alta calidad adecuadas a las necesidades de unos estudiantes cada vez más diversos. Este artículo analiza cómo un modelo de aprendizaje combinado ayuda a enfrentar estos desafíos, estimulando el entusiasmo de los estudiantes por el aprendizaje y mejorando la eficacia del proceso de enseñanza-aprendizaje a nivel de tasa de éxito, rendimiento académico y tasa de abandono.

La metodología utilizada, contrastando la opinión de la institución, de los estudiantes y del profesorado, permite evaluar la idoneidad del b-MOOC desde una triple perspectiva. Los resultados muestran que, más allá de su razón de ser inicial, los b-MOOC pueden "rentabilizarse" en el aula tradicional.

La satisfacción de los estudiantes es alta y el rendimiento académico mejora; al tiempo que los estudiantes se preparan mejor para un aprendizaje más autónomo, en el que las tecnologías de la información y comunicación juegan un papel destacado. Sin embargo, como señalan Nazarenko (2015) y Ramirez-Arellano, Acosta-Gonzaga, Bory-Reyes y Hernández-Simón (2018), la investigación sobre el aprendizaje en contextos combinados es muy limitada; por lo que sería deseable ampliarla a un mayor número de casos y estudiantes. Solo así se podrán asumir resultados fiables y generalizados.

Desde el punto de vista del profesorado, el nuevo modelo requiere una apuesta definitiva por el rol del docente como facilitador del aprendizaje. Dado que los b-MOOC dependen de las competencias digitales del profesorado, sería deseable más investigación para comprender la actitud del profesorado hacia la tecnología y sobre cómo ve su relevancia para el aprendizaje de los estudiantes (Kennedy et al., 2011). Paralelamente, también resultaría de interés analizar cómo la propia formación del profesorado puede ayudar a minimizar los obstáculos para el uso de modelos de aprendizaje combinados.

Finalmente, desde el punto de vista de las instituciones de educación superior, la experiencia expuesta en este artículo demuestra que los procesos de aprendizaje combinados son el motor definitivo para el cambio. Solo cuando el modelo pedagógico forma parte del plan estratégico de un centro, pueden sortearse las barreras a la innovación; incluidas las derivadas del coste económico, de la falta de tiempo y de recursos. El b-MOOC proporciona flexibilidad y un enfoque innovador en el proceso de enseñanza-aprendizaje que puede mejorar la calidad y relevancia de la educación superior. 
En los entornos de educación superior, los b-MOOC son una forma innovadora de aprendizaje, en el sentido de que brindan oportunidades a los estudiantes matriculados en la educación formal que comparten espacio con personas de todo el mundo. Sin embargo, estos dos grupos de estudiantes tienen objetivos diferentes cuando participan en los MOOC. Y, este un tema que necesita ser tratado con rigor. Los estudiantes matriculados en un grado tienen que aprobar una asignatura, mientras que los estudiantes inscritos en un MOOC están más interesados en el aprendizaje a lo largo de la vida (Yousef et al., 2015). Por ello, es importante valorar si esta diversidad en los objetivos puede ser un problema desde la perspectiva del diseño y gestión del curso.

\section{REFERENCIAS}

Baker, P. M. A., Bujaka, K. R., y DeMillo, R. (2012). The evolving university: Disruptive change and institutional innovation. Procedia Computer Science, 14, 330335. doi: https://doi.org/10.1016/j. procs.2012.10.037

Banerjee, A.V., y Duflo, E. (2014). Economics education in the digital age: the implications of online technologies and MOOCs: (Dis)Organization and success in an economics MOOC. American Economic Review: Papers \& Proceedings, 104(5), 514-518.

Baturay, H. M. (2015). An overview of the world of MOOCs. Procedia - Social and Behavioral Sciences, 174, 427433. doi: https://doi.org/10.1016/j. sbspro.2015.01.685

Bralić, A., y Divjak, B. (2018). Integrating MOOCs in traditionally taught courses: achieving learning outcomes with blended learning. International Journal of Educational Technology in Higher Education, 15(2), doi: https://doi. org/10.1186/s41239-017-0085-7

Caufield, M., Collier, A., y Halawa, S. (2013). Rethinking online community in MOOCs used for blended learning. Educause Review Online, 1-11. Recuperado de https://er.educause.edu/ articles/2013/10/rethinking-onlinecommunity-in-moocs-used-for-blendedlearning
Clarke, T. (2013). The advance of the MOOCs (massive open online courses): The impending globalisation of business education. Education + Training, 55(4/5), 403-413. doi: https://doi. org/10.1108/00400911311326036

Conijn, R., Van den Beemt, A., y Cuijpers, P. (2018). Predicting student performance in a blended MOOC. Journal of Computer Assisted Learning, 34, 615-628. doi: https://doi.org/10.1111/jcal.12270

Coppola, N. W., Hiltz, S. R., y Rotter, N. (2002). Becoming a virtual professor. Pedagogical roles and asynchronous learning networks. Journal of Management Information Systems, 18(4), 169-189. doi: https://doi.org/10.10 80/07421222.2002.11045703

Garrison, D. R., y Anderson, T. (2011). E-learning in the 21st Century: $A$ framework for research and practice. Londres: Routhledge/Falmer.

Garrison, D. R., y Vaughan, N. (2011). Blended learning in higher education. San Francisco: John Wiley \& Sons.

Gasevic, D., Kovanovic, V., Jokosimovic, S., y Simens, G. (2014). Where is research on massive open online courses headed? A data analysis of the MOOC research initiative. The International Review of Research in Open and Distance Learning, 15(5), 134-176. doi: https://doi. org/10.19173/irrodl.v15i5.1954 
Handal, B., MacNish, J., y Petocz, P. (2013). Academics adopting mobile devices: The zone of free movement. In M. Gosper, J. Hedberg y H. Carter (Eds.), Electric Dreams. Proceedings 3oth ASCILITE Conference, (350-361). Sydney: Macquarie University.

Hew, K., y Cheung, W. (2014). Students' and instructors' use of massive open online courses (MOOCs): Motivations and challenges. Educational Research Review, 12, 45-58. doi: 10.1016/j. edurev.2014.05.001.

Hollands, F., y Tirthali, D. (2014). Why Do Institutions Offer MOOCs? Online Learning, 18(3). Recuperado de https:// olj.onlinelearningconsortium.org/index. $\mathrm{php} / \mathrm{olj} / \mathrm{article} / \mathrm{view} / 464$

Holotescu, C., Grosseck, G., Cretu, V., y Naaji, A. (2014). Integrating MOOCs in blended courses. In I Rocean. Let's Build the Future through Learning Innovation! (vol. I). Paper presentado en 1oth International Scientific Conference on eLearning and software for Education, Bucharest, April 24-25 (pp. 237-242). Italia: Editura.

Hood, N., Littlejohn, A., y Milligan, C. (2015). Context counts: How learners' contexts influence learning in a MOOC. Computers y Education, 91, 83-91. doi: https://doi. org/10.1016/j.compedu.2015.10.019

Hoxby, C. M. (2014). The economics of online postsecondary education: MOOCs, nonselective education, and highly selective education. American Economic Review, 104(5), 528-533. doi: http:// dx.doi.org/10.1257/aer.104.5.528

Israel, M. J. (2015). Effectiveness of integrating MOOCs in traditional classrooms for undergraduate students. International Review of Research in Open and Distributed Learning, 16(5), 102-118. doi: https://doi.org/10.19173/ irrodl.v16i5.2222

Jansen, D., y Schuwer, R. (2015). Institutional MOOC strategies in Europe Status report based on a mapping survey conducted in
October - December 2014. Recuperado de http://www.eadtu.eu/documents/ Publications/OEenM/Institutional MOOC strategies in Europe.pdf

Kennedy, G., Jones, D., Chambers, D., y Peacock, J. (2011). Understanding the reasons academics use - and don't use - endorsed and unendorsed learning technologies. En Proceedings of ASCILITE - Australian Society for Computers in Learning in Tertiary Education Annual Conference 2011 (pp. 688-701). Australasian Society for Computers in Learning in Tertiary Education. Recuperado de https://www. learntechlib.org/p/43613/

Kovanović, V., Joksimović, S., Gašević, D., Siemens, G., y Hatala, M. (2015). What public media reveals about MOOCs: A systematic analysis of news reports. British Journal of Educational Technology, 46(3), 510-527. doi: https:// doi.org/10.1111/bjet.12277

Liyanagunawardena, T. R., Lundqvist, K. Ø., y Williams, S. A. (2015). Who are with us: MOOC learners on a FutureLearn course. British Journal of Educational Technology, 46, 557-569.

Loya, A., Gopal, A., Shukla, I., Jermann, P., y Tormey, R. (2015). Conscientious behaviour, flexibility and learning in massive open on-line courses. Procedia - Social and Behavioral Sciences, 191, 519-525. doi: https://doi.org/10.1016/j. sbspro.2015.04.686

Margaryan, A., Bianco, M., y Littlejohn, A. (2015). Instructional quality of Massive Open Online Courses (MOOCs). Computers y Education, 80, 77-83. doi: $\quad \underline{\text { http://dx.doi.org/10.1016/j. }}$ compedu.2014.08.0050360-1315

Mirriahi, N., Alonzo, D., y Fox, B. (2015). A blended learning framework for curriculum design and professional development. Research in Learning Technology, 23, doi: http://dx.doi. org/10.3402/rlt.v23.28451 
Mirriahi, N., Alonzo, D., McIntyre, S., Kligyte, G., y Fox, B. (2015). Blended learning innovations: Leadership and change in one Australian institution. International Journal of Education and Development using Information and Communication Technology, 1 (1), 4-16.

Moskal, P., Dziuban, C., y Hartman, J. (2013). Blended learning: A dangerous idea? Internet \& Higher Education, 18, 15-23. doi: http://dx.doi.org/10.1016/j. iheduc.2012.12.001

Nazarenko, A. L. (2015). Blended learning vs traditional learning: What works? (a case study research). Procedia - Social and Behavioral Sciences, 20o(22), 77-82. doi: https://doi.org/10.1016/j. sbspro.2015.08.018

Norman, G. (2010). Likert scales, levels of measurement and the "laws" of statistics. Advances in Health Science Education, 15, 625-632. doi: 10.1007/s10459-0109222-y.

Osuna-Acedo, S., Marta-Lazo, C., y FrauMeigs, D. (2018). From sMOOC to tMOOC, learning towards professional transference. ECO European Project. Comunicar, 55, 105-114. doi: https://doi. org/10.3916/C55-2018-10

Owston, R., York, D., y Murtha, S. (2013). Student perceptions and achievement in a university blended learning strategic initiative. Internet \& Higher Education, 18, 38-46. doi: https://doi.org/10.1016/j. iheduc.2012.12.003

Ramirez-Arellano, A., Acosta-Gonzaga, E., Bory-Reyes J., y Hernández-Simón, L. M. (2018). Factors affecting student learning performance: A causal model in higher blended education. Journal of Computed Assisted Learning, 34, 807-815. doi: https://doi.org/10.1111/jcal.12289

Ronkowitz, K., y Ronkowitz, L. C. (2015). MOOCs: Evolution and revolution. In E. McKay y J. Lenarcic Eds.), Macro-level learning through massive open online courses (MOOCs), (183-211). Hershey: IGI Global.

Smith, B., y Eng, M. (2013). MOOCs: A learning journey. In S. K. S. Cheung, J. Fong, W. Fong, F. L. Wang, y L. F. Kwok (Eds.), Hybrid Learning and Continuing Education, (244-255). Berlin: Springer Berlin Heidelberg.

Torrisi-Steele, G., y Drew, S. (2013). The literature landscape of blended learning in higher education: the need for better understanding of academic blended practice. International Journal for Academic Development, 18(4), 371-383. doi: https://doi. org/10.1080/1360144X.2013.786720

Tselios, N., Daskalakis, S., y Papadopoulou, M. (2011). Assessing the acceptance of a blended learning university course. Educational Technology y Society, 14(2), 224-235.

Yousef, A. M. F., Chatti, M. A., y Schroeder, U. (2014). Video-based learning: A critical analysis of the research published in 2003-2013 and future visions. En M. Marquand, S. White y M. A. Lakhanie, eLmL 2014. The Sixth International Conference on Mobile, Hybrid and Online Learning. Barcelona, Marzo 23-27, (pp. 112-119). Barcelona: Iaria.

Yousef, A., Chatti, M., Schroeder, U., y Wosnitza, M. (2015). A usability evaluation of a blended MOOC environment: An experimental case study. The International Review of Research in Open and Distributed Learning, 16(2). Recuperado de http:// www.irrodl.org/index.php/irrodl/article/ view $/ 2032 / 3270$

Yuan, L., Powell, S., y Olivier, B. (2014). Beyond MOOCs: Sustainable online learning in institutions. Bolton: CETIS. Recuperadodehttps://www.researchgate. net/publication/265297773 Beyond MOOCs Sustainable Online Learning in Institutions 
Zhang, X., Huang, X., Wang, F., y Cao, X. (2018). Research on MOOC-based blended learning of programming language course. Paper presentado en la International Conference on Humanities and Advanced Education Technology (ICHAET 2018) Guangzhou (China),
Agosto 19-20. (pp. 586-591). doi: 10.12783/dtssehs/ichae2018/25707.

Zhou, M. (2016). Chinese university students' acceptance of MOOCs: A self-determination perspective. Computers y Education, 92-93, 194203. doi: http://dx.doi.org/10.1016/j. compedu.2015.10.012

\section{PERFIL ACADÉMICO Y PROFESIONAL DE LAS AUTORAS}

María-Arántzazu Vidal-Blasco es Doctora en Administración y Dirección de Empresas (Universitat Rovira i Virgili, España, 2001). Profesora titular de Universidad del Departamento de Gestión de Empresas de la Universitat Rovira i Virgili. Ha colaborado en proyectos de investigación sobre e-learning y educación superior, sobre desarrollo de competencias en la era digital, networking, y sobre inteligencia emocional y su relación con el emprendimiento.

E-mail: mariaarantzazu.vidal@urv.cat

Dirección:

Facultad de Ciencias Económicas y Empresariales Universitat Rovira i Virgili

Av. de la Universitat, 2

43204 Reus -Tarragona (España)

Teresa Torres-Coronas es Doctora en Administración y Dirección de Empresas (Universitat Rovira i Virgili, España, 2000). Profesora titular de Universidad del Departamento de Gestión de Empresas de la Universitat Rovira i Virgili. Sus campos de investigación se enfocan al estudio del mercado laboral y el encaje competencial entre demanda y oferta, así como a analizar el papel de las TIC en los procesos de enseñanza-aprendizaje en el ámbito de la educación superior.

E-mail: teresa.torres@urv.cat

Dirección:

Escuela Técnica Superior de Ingeniería

Universitat Rovira i Virgili

Av. Països Catalans, 26

Tarragona (España)

Fecha de recepción del artículo: 21/03/2019

Fecha de aceptación del artículo: 12/04/2019

Fecha de maquetación: 23/05/2019 